\title{
Highly Selective Metal-Organic Framework Textile Humidity Sensor
}

\author{
Sakandar Rauf, Mani Teja Vijjapu, Miguel A. Andrés, Ignacio Gascón, Olivier Roubeau, \\ Mohamed Eddaoudi, and Khaled Nabil Salama*
}

Cite This: ACS Appl. Mater. Interfaces 2020, 12, 29999-30006

Read Online

ABSTRACT: The increase in demand and popularity of smart textiles brings new and innovative ideas to develop a diverse range of textile-based devices for our daily life applications. Smart textilebased sensors (TEX sensors) become attractive due to the potential to replace current solid-state sensor devices with flexible and wearable devices. We have developed a smart textile sensor for humidity detection using a metal-organic framework (MOF) as an active thin-film layer. We show for the first time the use of the Langmuir-Blodgett (LB) technique for the deposition of a MIL96(Al) MOF thin film directly onto the fabrics containing interdigitated textile electrodes for the fabrication of a highly selective humidity sensor. The humidity sensors were made from

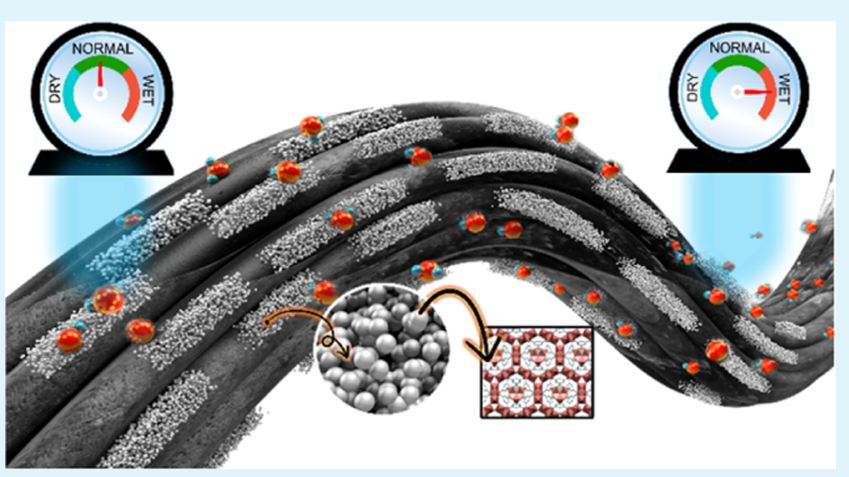
two different types of textiles, namely, linen and cotton, with the linen-based sensor giving the best response due to better coverage of MOF. The TEX sensor showed a reproducible response after multiple cycles of measurements. After 3 weeks of storage, the sensor showed a moderate decrease in response. Moreover, TEX sensors showed a high level of selectivity for the detection of water vapors in the presence of several volatile organic compounds (VOCs). Interestingly, the selectivity is superior to some of the previously reported MOF-coated solid-state interdigitated electrode devices and textile sensors. The method herein described is generic and can be extended to other textiles and coating materials for the detection of toxic gases and vapors.

KEYWORDS: metal-organic framework (MOF), MIL-96(Al), thin film, textile sensor, humidity sensor, interdigitated electrodes (IDEs), Langmuir-Blodgett (LB) films

\section{INTRODUCTION}

Smart textiles have emerged as a new market due to the growing interest in flexible and wearable electronic devices for various applications such as measurement of human physiological signals of pressure and temperature, energy storage and harvesting, gas sensors, and biosensors. ${ }^{1-5}$ The inclusion of Internet of Things (IoT) technology in smart textiles is another crucial factor that has been contributing to the growth in the smart textile market. Smart textiles are expected to create the fourth industrial revolution for the global textile and fashion industry worth U.S. $\$ 130$ billion by $2025 .^{6}$ Smart textiles extend the functionality and usefulness of the everyday fabric by combining electronics and smart materials that have a diverse spectrum of functionalities. ${ }^{7}$ The choice in the inclusion of a wide variety of nanomaterials, such as metalorganic frameworks (MOFs) with various functions, is an excellent opportunity to extend the application landscape of smart textiles with enhanced performance. These materials combine the highly regular and well-defined pore shape of inorganic porous materials (e.g., zeolites) with the high chemical versatility of organic linkers. All of these properties account for the selective adsorption and detection of gases and vapors. $^{8}$
MOFs have been used extensively for the fabrication of chemical sensors, biosensors, and gas sensors in combination with solid-state devices. ${ }^{9-13}$ Despite the fact that the combination of MOFs and textiles has been utilized for a wide range of other applications such as UV protection, ${ }^{14,15}$ antibacterial coating, ${ }^{16}$ protective warfare agent clothing, ${ }^{17}$ and catalysis, ${ }^{18}$ there are fewer reports on gas sensors made from a combination of textiles and MOFs. ${ }^{19,20}$ In most cases, the methods for making MOF-textile functional materials involve in situ synthesis of the MOF onto the fabric, ${ }^{19}$ spray coating of MOF directly onto the fabric, ${ }^{17}$ solvent-free hot-pressing technology, ${ }^{15}$ and direct immobilization of MOFs onto the fabric. ${ }^{21}$ To date, the Langmuir-Blodgett (LB) film method has not yet been tested for the deposition of MOFs onto textile fabrics and subsequently used for applications such as sensors.

Received: April 23, 2020

Accepted: June 9, 2020

Published: June 9, 2020 
The LB technique is a well-established method for the preparation of thin films at the air-water interface. ${ }^{22,23}$ Compared to other methods used to deposit MOF thin films, the LB technique does not require previous functionalization of the surface and minute amounts of material are used. Moreover, the film thickness can be precisely controlled as compared to other coating methods, ${ }^{24}$ and it can be as thin as one monolayer of nanoparticles. The LB method has been used so far for different types of MOF coatings mainly onto solid-state devices. $^{24-27}$

The measurement of the concentration of water vapors (humidity) is widely used in many sectors related to our daily life, such as building automation, pharmaceuticals, hospitals, chemical industry, and the food industry. ${ }^{28}$ Moreover, extreme conditions of humidity have adverse effects on human health and are linked to many diseases. ${ }^{29}$ Different types of humidity sensors have been developed based on either solid-state devices, ${ }^{24,30-40}$ flexible substrates, ${ }^{4-47}$ inkjet printed textile, ${ }^{48}$ or textile fiber composite materials. ${ }^{3,49-55}$ Most of these studies demonstrated the sensitivity of the sensor; however, only a few of these discussed the selectivity of the sensor, which is equally important. Moreover, in the case of textilebased humidity sensors, to the best of our knowledge, there is no report describing the selectivity of the textile sensors in the presence of interferences such as volatile organic compounds (VOCs). Therefore, not only sensitive but also selective textile humidity sensors are highly desirable that can operate in various environments under different conditions.

Herein, we show, for the first time, a highly selective MOFcoated textile sensor prepared using the LB technique and demonstrate its application as a chemicapacitive humidity sensor. MIL-96(Al) MOF was chosen for coating a thin film directly on the fabric containing interdigitated conductive thread electrodes as an active layer of humidity sensor due to its thermal stability along with high water adsorption capacity and moisture stability, ${ }^{24,56}$ and the fact that we have previously shown that high-quality films can be obtained using nanoparticles (NPs) of this material. ${ }^{24,26}$ Our results showed that the MOF-coated fabric sensor (TEX sensor) has higher selectivity toward water in the presence of other volatile organic compounds (VOCs), which is similar or better in many cases as compared to other previously reported solidstate sensors. $^{24,40}$ These results show the first demonstration of a highly selective textile humidity sensor and are highly valuable as many of the reported textile humidity sensors may exhibit cross-sensitivity to VOCs. The developed MOF-coated interdigitated textile sensor demonstrates a generic method of the incorporation of MOF thin films using the LB method, which opens up the possibilities to develop several other textile-based sensors by varying the type of MOF used for coating.

\section{EXPERIMENTAL SECTION}

Nanoparticles of MIL-96(Al) $(\sim 200 \mathrm{~nm}$ in size $)$ were used in these studies. Details of the synthesis and characterization of MIL-96(Al) are included in the Supporting Information (Figure S1). To fabricate the interdigitated textile electrodes, we first selected two of the most common commercially available fabrics, namely, cotton and linen, that are widely used in the textile industry for the production of clothes or garments. A conductive thread (Liberator 40) was used for the fabrication of interdigitated electrodes onto the fabrics. Liberator 40 Vectran silver-coated conductive thread was purchased from Amazon US. The conductive thread was stitched following the handstitched embroidery method, which involves tracing the design of the interdigitated electrodes onto the fabric (Figure S2, Supporting Information). Custom-built holders of transparent poly(methylmethacrylate) (PMMA) sheets were fabricated using a VLS 3.5 Desktop laser platform equipped with a $\mathrm{CO}_{2}$ laser. Circular holders were used for holding the interdigitated textile electrodes during the Langmuir-Blodgett film deposition process. LangmuirBlodgett film formation of MIL-96(Al) was carried out using a commercially available KSV-NIMA trough, model 2000-System 3, with dimensions $775 \times 120 \mathrm{~mm}^{2}$ and a symmetrical double-barrier system. Briefly, previous studies using dynamic light scattering (DLS), surface pressure-area $(\pi-A)$ and surface potential-area $(\Delta V-A)$ isotherms, and Brewster angle microscopy (BAM) have shown that homogeneous and compact MIL-96(Al) monolayer films can be obtained at the air-water interface using dispersions in chloroform prepared by ultrasonication (MOF concentration $0.2 \mathrm{mg} / \mathrm{mL}$ ). ${ }^{26}$ Moreover, these monolayers can be effectively transferred onto substrates of different natures (glass, quartz, mica, QCM disks, $\mathrm{Si}(100)$, and $\mathrm{Si} / \mathrm{SiO}_{2}$ with $\mathrm{Au}$ IDEs at a surface pressure of $30 \mathrm{mN} /$ $\mathrm{m}){ }^{24,26}$ Before compressing the film, the water surface was carefully cleaned to remove any material released from the clothes/fibers into the water. For that purpose, barriers were closed down to $3 \mathrm{~cm}$ distance, and the surface between the barriers was cleaned by mild surface-touch vacuuming. This process was repeated until a surface pressure increase lower than $0.1 \mathrm{mN} / \mathrm{m}$ was observed. Typically, 8 $\mathrm{mL}$ of MIL-96(Al) suspension was spread drop by drop on the water surface using a Hamilton microsyringe (initial area before compression about $500 \mathrm{~cm}^{2} / \mathrm{mg}$ of MOF) and then, after solvent evaporation, films were compressed at constant speed $\left(6 \mathrm{~cm}^{2} / \mathrm{min}\right)$ until the desired transfer surface pressure $(30 \mathrm{mN} / \mathrm{m})$ was achieved. Langmuir-Blodgett films were deposited onto fabrics fixed into PMMA holders initially immersed in the water subphase, and vertical dipping was performed at $1 \mathrm{~mm} / \mathrm{min}$. Langmuir-Schaefer (LS) films were prepared by holding the textile parallel to the surface. The textile holder was approached to the water surface at a vertical speed of 1 $\mathrm{mm} / \mathrm{min}$. After touching the surface, the holder was raised using a vertical speed of $10 \mathrm{~mm} / \mathrm{min}$. Reverse Langmuir-Schaefer (RLS) films were prepared in a similar manner, but the textile was initially immersed in the subphase. After reaching the desired surface pressure $(30 \mathrm{mN} / \mathrm{m})$, the holder was raised at a vertical rate of $1 \mathrm{~mm} / \mathrm{min}$. All LB/LS/RLS films were allowed to dry in the air overnight and then stored in a desiccator.

For the characterization of MOF deposition onto interdigitated textile electrodes, a Quanta 3D FEG SEM/FIB microscope was used with an accelerating voltage of $10 \mathrm{kV}$. A $5 \mathrm{~nm}$ conductive layer of iridium was sputtered on all samples before the inspection. To test the sensing performance of the MOF-coated interdigitated textile devices, test probes with alligator cables were designed and placed into the measuring chamber of a custom-built setup (Figure S3, Supporting Information), which contains a thermostatic bath, mass-flow controllers, and a Keysight E4980A LCR meter. All of the capacitance measurements were performed at $100 \mathrm{kHz} .{ }^{24}$ Nitrogen was used as a carrier gas and was flown through glass bubblers filled with deionized water and different organic solvents to generate vapors (wet current). The bubbler sources are immersed in an F12-MA Julabo thermostatic bath to have control over temperature. The wet current was mixed with a dry current of nitrogen as dilution and introduced into the measurement chamber. The concentration of water vapor or volatile organic compound vapors (VOCs) introduced into the cell was calculated as described previously by some of $\mathrm{us}^{24}$ and expressed in parts per million in volume (ppm). A constant flow of $200 \mathrm{sccm}$ of gas was maintained in all experiments. In every experiment, water vapors or VOCs were introduced for $3 \mathrm{~min}$ inside the chamber during transient analysis, and the change in capacitance was measured. Before starting every experiment, all MOF-coated textile sensors were heated for $10 \mathrm{~h}$ at $100{ }^{\circ} \mathrm{C}$ to desorb the water captured by MOF particles. While evaluating the sensor response, the change in capacitance with time at different humidity contents was measured using nitrogen to purge the sensor after each concentration step. All of the measurements were performed at $22{ }^{\circ} \mathrm{C}$ unless otherwise stated. 
(a)

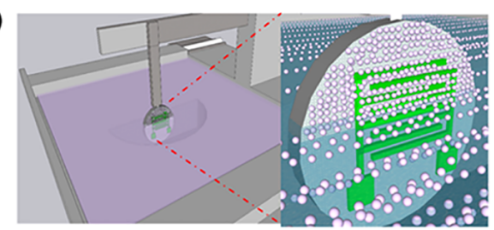

(c)

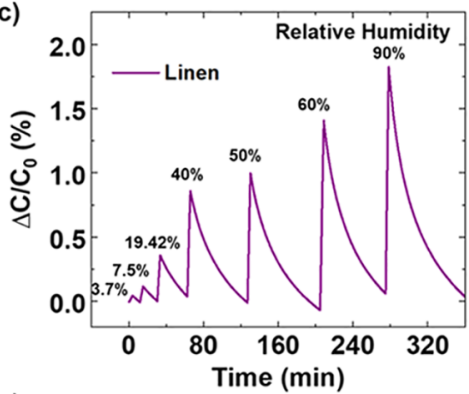

(e)

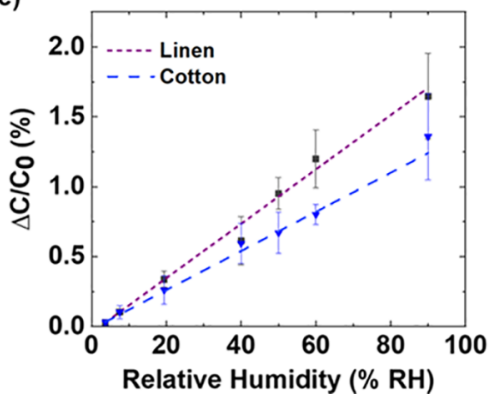

(b)

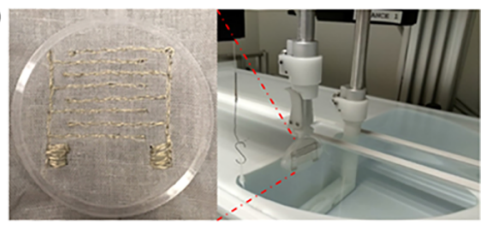

(d)

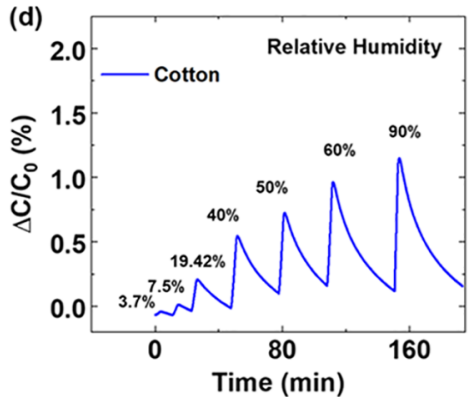

(f)

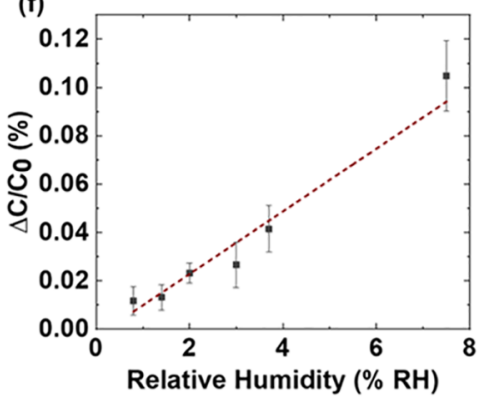

Figure 1. (a, b) Scheme and photograph of the setup used for Langmuir-Blodgett (LB) deposition. (c, d) Response curve of the linen and cotton TEX sensors in the presence of humidity from 3.7 to $90 \%$. (e) Comparison of the linen and cotton TEX sensor responsivities. Both types of sensors showed a linear response. (f) Calibration curve of the linen TEX sensor in the low-relative-humidity range (from 0.71 to $7.5 \%$ ). Error bars correspond to the standard deviation from the measurement of three different samples. Relative humidity was measured at $22{ }^{\circ} \mathrm{C}$.

\section{RESULTS AND DISCUSSION}

\subsection{MOF-Coated Interdigitated Textile Electrodes for} Humidity Detection. To fabricate the interdigitated textile electrodes onto the fabric, the conductive fiber Liberator 40 was stitched as an interdigitated electrode pattern into two different types of fabrics, namely, cotton and linen. Fixed step size and number of fingers of these electrodes were maintained uniformly for all of the fabricated sensors (Figure S2, Supporting Information). Figure 1a,b shows the LangmuirBlodgett coating setup for the deposition of MIL-96(Al) particles onto the fabric containing interdigitated conductive thread electrodes. The interdigitated textile electrode fabric was first fixed into a custom-built PMMA circular frame so that the fabric remains flat during the deposition of MOF particles (Figures 1a,b and S2). The transfer of one layer was possible using LB and Langmuir-Schaefer/reverse Langmuir-Schaefer (LS/RLS) transfer on both linen and cotton fabrics. We also explored the feasibility of multilayer deposition using the LB method or RLS transfer, but a part of the previously deposited layer was detached from the cloth when the holder was immersed into the subphase for next layer deposition. This could be seen with the naked eye from dust appearing in the water surface, which had the same appearance as the water surface after spreading the MOF suspension. In addition, a large increase in surface pressure was observed for the clean subphase even when barriers were not fully closed. From preliminary sensing experiments, higher responses were obtained using vertical LB deposition. Besides, LB deposition accounts for more straightforward automation for film preparation in comparison to LS deposition, where the dipping process has to be precisely controlled. Hence, we decided to perform all of the studies using vertical LB deposition of one MOF layer.

The MOF-coated sensors (TEX sensor) exhibited a baseline capacitance $(\sim 1.97 \mathrm{pF})$ due to the dielectric nature of MIL96(Al) MOF particles (Figure S4, Supporting Information). Water has a very high dielectric constant $(\sim 80),{ }^{57}$ and the MIL-96(Al) MOF particles have excellent affinity for water. ${ }^{24}$ Hence, we tested our devices in the presence of humidity, ranging from values of 3.7 to $90 \%$ of relative humidity $(\mathrm{RH})$. To understand the nature of the fabric effect in the sensing performance, we compared the sensors made of two fabrics, linen and cotton. TEX sensors using linen and cotton fabrics revealed a significant change in capacitance in the presence of water, as shown in Figure 1c,d, respectively. The capacitance of sensors increased proportionally with the increase in humidity. This is because the dielectric constant of the active medium in the sensor proportionally increases with the increase in humidity, which results in the increase of the sensor's capacitance. ${ }^{30}$ Interestingly, the devices were regenerated in the presence of nitrogen, and no heating or vacuum was required to recover the device after exposure. In the absence of the MOF layer, the charge storage contribution of the pristine textile fabric is negligible, resulting in extremely low baseline capacitance values $(<1 \mathrm{fF})$, which is consistent with the previously reported textile-based wearable capacitors. ${ }^{58}$

The higher responsivity was observed in the case of the linen TEX sensor, as shown in Figure 1c,e. Moreover, linen-based TEX sensors exhibited a linear increase in response with the 

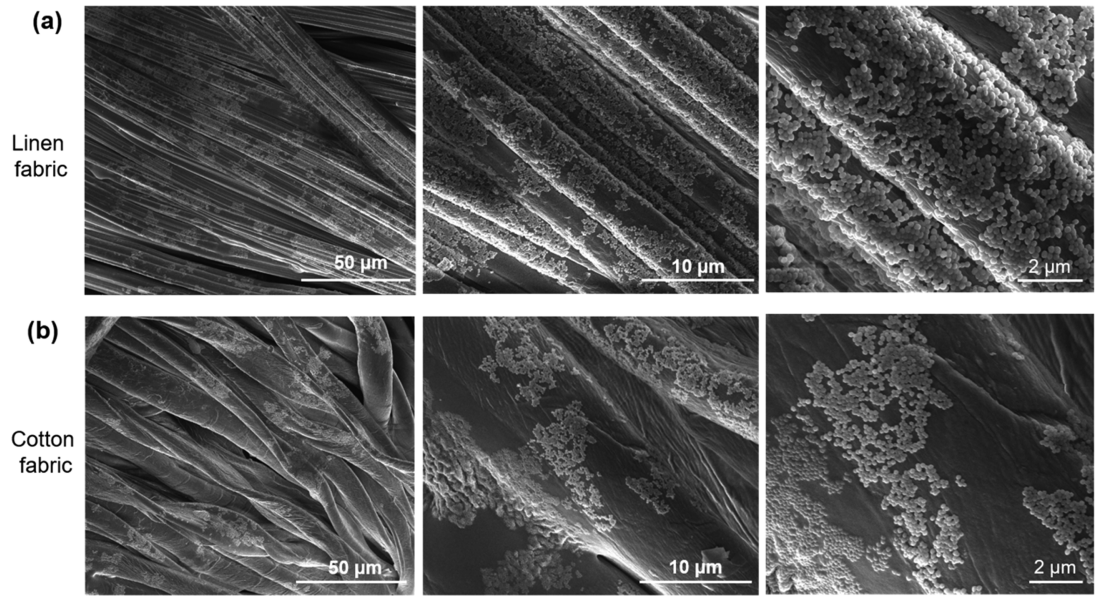

Figure 2. Representative scanning electron microscopy (SEM) images of (a) linen- and (b) cotton-based textile sensors coated with a MIL-96(Al) LB film. Higher magnification images from left to right.

increase in the concentration of water vapor, even with concentrations as low as $0.71 \%$ of relative humidity (Figures $1 \mathrm{f}$ and S5). The limit of detection and sensitivities in the case of the linen TEX sensors were found to be $0.71 \% \mathrm{RH}$ and 0.02 $(100 \cdot \Delta C / C)$ per percent of $\mathrm{RH}$, respectively (Figure 1f). In the case of the cotton TEX sensor, the limit of detection and sensitivities were $3.7 \% \mathrm{RH}$ and $0.013(100 \cdot \Delta C / C)$ per percent of $\mathrm{RH}$, respectively (Figure 1e).

To understand these results, we performed scanning electron microscopy (SEM) studies, as depicted in Figure 2, which revealed that the coverage of the MOF is better in the case of linen fabric, thus accounting for its higher responses. Moreover, the linen threads in the textile are thinner and better arranged than cotton, hence producing less perturbation to the LB film (Figure 2). In addition, SEM images also showed that dense MOF particle deposits tend to build up at the junction points or the space between the turns of different fibers of Liberator 40 along the fiber bundles (Figure 3a,b). It is important to note that in the case of linen and cotton fabrics,

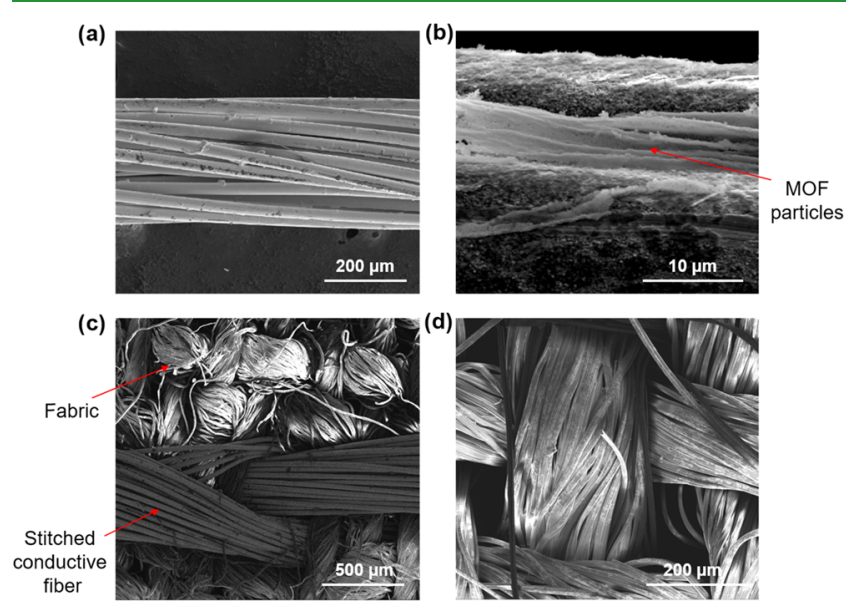

Figure 3. Scanning electron microscopy (SEM) studies. (a) Liberator 40 conductive fiber used for LB film deposition studies. (b) After the deposition of MOF. The red arrows indicate the accumulation of MOF particles deposited at the junctions or twist of the fibers inside Liberator 40 fiber bundles. (c) Shows the conductive fiber stitched into the fabric. (d) Shows the coating of MIL-96(Al) LB onto the linen-fabric fibers. the MOF film is not continuous as compared to the LB films deposited onto flat solid-state devices. ${ }^{24,26}$ This can be explained by the fact that the surface of fabrics is not uniform, and the undulating surface is due to the knitting of several single fibers together in a three-dimensional arrangement (Figure $3 c, d$ ). The uneven surface of fabrics causes slight discontinuities in the MOF film as compared to the planar solid-state devices. Despite this, the TEX sensors are still highly sensitive toward water vapors. Therefore, we propose that the sensor signal is due to the synergetic combination of the fabric and MOF particles, as the sensor response was also found to be dependent on the nature of the fabric.

3.2. Reproducibility and Stability Studies of the Textile Sensor. One of the critical parameters to develop gas sensors is the reproducibility of the sensor and its stability after storage. For further studies, we used linen-based TEX sensors due to their better performance as compared to the cotton TEX sensors. Linen TEX sensor showed a similar response after multiple cycles of operation (Figure 4a) at room temperature, which is essential to deploy these types of devices as real-time sensors. For the reproducibility tests, TEX sensors were exposed to the same concentration of humidity ( 5000 $\mathrm{ppm}$ ) for multiple times, and after each exposure, devices were recovered by purging with nitrogen. We have also evaluated
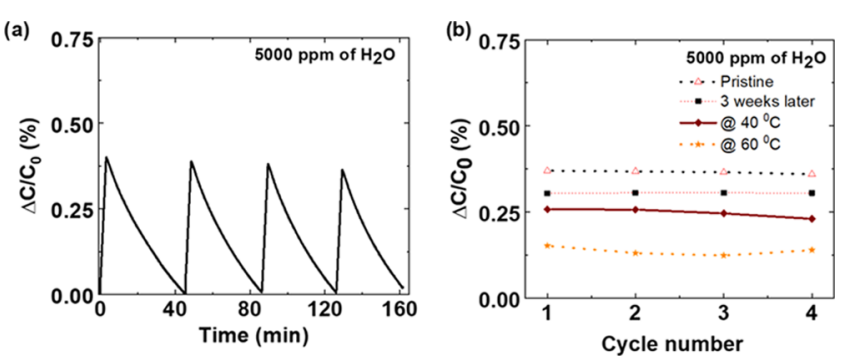

Figure 4. (a) Capacitive response curve for recycling experiments performed on linen TEX sensor at $5000 \mathrm{ppm}$ of water vapor at $22{ }^{\circ} \mathrm{C}$ (19.42\% relative humidity at the same temperature). (b) Normalized capacitive responses for water vapor $(5000 \mathrm{ppm})$ recycling experiments at 40 and $60{ }^{\circ} \mathrm{C}$. For stability studies, the TEX sensor was stored inside a Petri dish under ambient air at room temperature (22 ${ }^{\circ} \mathrm{C}$ ) for 3 weeks. The sensor response was compared to that of a pristine sample. For comparison purposes, a plot for a pristine sample is included. 
the sensing response at different temperatures. For this purpose, the whole chamber in which devices were tested was placed on a hotplate (Figure S3, Supporting Information), which maintained the required temperatures inside the test chamber (recorded by an LM235 temperature sensor). Responsivity decreased with the increase in temperature (Figures $4 \mathrm{~b}$ and S6), as expected by the general trend of decrease in sorption capacities of MOF particles at higher temperatures. ${ }^{11}$ Moreover, the recovery times were drastically decreased with the increase in temperature. Such eased regeneration of the samples with increased temperature is expected because of the increase in the vapor pressure of water. Finally, long-term stability was evaluated. For this purpose, the response of a TEX sensor stored inside a Petri dish under ambient air at room temperature $\left(22{ }^{\circ} \mathrm{C}\right)$ for 3 weeks was compared to that of a pristine sample. Interestingly, the aged TEX sensor only showed a moderate decrease in the response (16\%) as compared to the as-prepared TEX sensor (Figure $4 \mathrm{~b})$, indicating the stability of the sensor. In a previous study, we have shown that the surface barrier phenomenon plays an important role in the adsorption properties of MIL-96(Al) NP films. ${ }^{26}$ The outer surface of the NPs undergoes changes upon exposure to ambient conditions, which results in the formation of surface barriers. ${ }^{59}$ These barriers make access of water molecules more difficult from the air to the MOF pores. Nevertheless, the response of the sensor after 3 weeks, shows a decrease of only $16 \%$ in sensitivity even though it was not stored in special conditions. Hence, the textile humidity sensor developed in these studies can be stored and reused, which is an essential feature for gas sensors.

3.3. Selectivity Studies of the TEX Sensor. Selectivity is a crucial parameter for the characterization of any type of sensors. In the case of MOF materials, it is challenging to achieve a high level of specificity by the shape and size of the MOF alone. ${ }^{60}$ However, installing sophisticated recognition elements might be able to extend the selectivity of the MOFbased sensors for various analytes. ${ }^{60,61}$ MIL-96(Al) MOF shows a high affinity toward water and methanol vapors. ${ }^{56}$ Due to this high adsorption of water and methanol, some of us have observed that the MIL-96 MOF-coated interdigitated electrode-based solid-state sensor selectivity toward water vapors in the presence of methanol and other VOCs was not sufficient. To address this issue, an additional coating of Parylene $\mathrm{C}$ film was applied to detect water vapors in the presence of methanol selectively. ${ }^{24}$ Importantly, MIL-96(Al) LB-based TEX sensors have shown remarkable selectivity toward humidity in the presence of other volatile organic compounds (VOCs), including methanol (Figure 5). No additional coating was required to enhance the selectivity of the textile sensor, as in the case of the solid-state sensor. ${ }^{24}$ We believe that the improved selectivity of the TEX sensor may be due to the synergistic combination of the fabric and the discontinuities in the MOF layer, resulting in lower combined adsorption of VOCs and sufficient water adsorption, thereby giving a measurable and different enough signal for water vapors to be selectively detected. Interestingly, in the case of textile humidity sensors, to the best of our knowledge, there are no selectivity studies reported, such as those accounting for the effect of VOCs in the response, which is highly crucial for the evaluation of the performance of gas sensors. It is important to note that the concentration values of the VOCs used in our studies (5000 ppm) are much higher than the National Institute of Occupational Safety and Health's

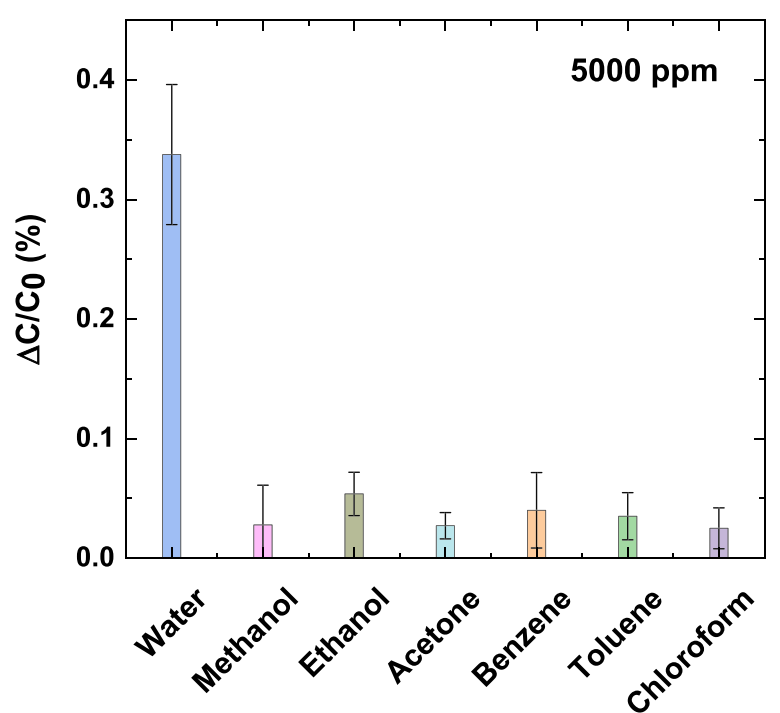

Figure 5. Selectivity of linen TEX sensors toward water vapor at 5000 ppm (19.42\% relative humidity) and negligible responsivity in the presence of other volatile organic compound (VOC) interferences (5000 ppm for each analyte). Error bars correspond to the standard deviation from the measurement of three different samples.

recommended exposure limit (NIOSH REL) ${ }^{62}$ values, which range from 2 to $1000 \mathrm{ppm}$ for these VOCs, therefore indicating that TEX sensor is highly selective even at higher concentrations of VOCs. We rate our sensor as superior in terms of selectivity as compared to other previously reported textile humidity sensors. Table S1 (Supporting Information) shows a comparison of our sensor with other types of humidity sensors reported in the literature. The sensitivity of our humidity sensor matches or is comparable to many of the humidity sensors, both solid-state and textile-based. However, in terms of selectivity, our sensor is highly superior to other textile-based humidity sensors.

\section{CONCLUSIONS}

In this work, we have demonstrated the feasibility of the deposition of a thin layer of MIL-96 (Al) MOF particles onto interdigitated electrode-based fabrics using the LangmuirBlodgett method. The TEX sensors obtained by directly coating the MOF LB layer onto the interdigitated textile electrodes were highly selective toward the detection of humidity in the presence of several VOCs. The linen TEX sensor exhibited $0.6 \mathrm{fF}$ per \%RH sensitivity with around $0.71 \%$ $\mathrm{RH}$ limit of detection. The type of textile proved to be relevant to obtain optimum performance of the sensor associated with an optimal deposition of the MOF particle film. Moreover, the TEX sensors were stable over several sensing cycles and, when stored for 3 weeks, only showed a moderate change in the response while maintaining the sensor properties. Overall, we showed a generic method for the development of MOF-based textile sensors, which opens up the possibility of developing several other sensors by changing the type of MOF material sensitive to a particular analyte. This is a crucial step to develop smart textiles and safety suits for the personnel working in laboratories, mines, petrochemical industries, and such other vulnerable industries. 


\section{ASSOCIATED CONTENT}

\section{(s) Supporting Information}

The Supporting Information is available free of charge at https://pubs.acs.org/doi/10.1021/acsami.0c07532.

MOF synthesis and characterization; experimental setup; capacitive response curves for linen textile; recycling experiments performed on linen TEX sensor at $5000 \mathrm{ppm}$ of water vapor at different temperatures; and comparison of different sensors (Table S1) (PDF)

\section{AUTHOR INFORMATION}

\section{Corresponding Author}

Khaled Nabil Salama - Sensors Lab, Advanced Membranes \& Porous Materials Center (AMPMC), Computer, Electrical and Mathematical Sciences and Engineering (CEMSE) Division, King Abdullah University of Science and Technology (KAUST), Thuwal 23955-6900, Kingdom of Saudi Arabia; (1) orcid.org/ 0000-0001-7742-1282; Email: khaled.salama@kaust.edu.sa

\section{Authors}

Sakandar Rauf - Sensors Lab, Advanced Membranes \& Porous Materials Center (AMPMC), Computer, Electrical and Mathematical Sciences and Engineering (CEMSE) Division, King Abdullah University of Science and Technology (KAUST), Thuwal 23955-6900, Kingdom of Saudi Arabia; (1) orcid.org/ 0000-0001-6978-3312

Mani Teja Vijjapu - Sensors Lab, Advanced Membranes \& Porous Materials Center (AMPMC), Computer, Electrical and Mathematical Sciences and Engineering (CEMSE) Division, King Abdullah University of Science and Technology (KAUST), Thuwal 23955-6900, Kingdom of Saudi Arabia; —orcid.org/ 0000-0002-1386-0689

Miguel A. Andrés - Departamento de Quimica Fisica and Instituto de Nanociencia de Aragón (INA), Universidad de Zaragoza, 50009 Zaragoza, Spain; Instituto de Ciencia de Materiales de Aragón (ICMA), CSIC and Universidad de Zaragoza, 50009 Zaragoza, Spain; 다이.org/0000-00033691-3437

Ignacio Gascón - Departamento de Química Fisica and Instituto de Nanociencia de Aragón (INA), Universidad de Zaragoza, 50009 Zaragoza, Spain; Instituto de Ciencia de Materiales de Aragón (ICMA), CSIC and Universidad de Zaragoza, 50009 Zaragoza, Spain; 이잉.org/0000-00023492-6456

Olivier Roubeau - Instituto de Ciencia de Materiales de Aragón (ICMA), CSIC and Universidad de Zaragoza, 50009 Zaragoza, Spain; 이이이.org/0000-0003-2095-5843

Mohamed Eddaoudi - Functional Materials Design, Discovery \& Development Research Group (FMD3), Advanced Membranes \& Porous Materials Center, Division of Physical Sciences and Engineering, King Abdullah University of Science and Technology (KAUST), Thuwal 23955-6900, Kingdom of Saudi Arabia; ○ orcid.org/0000-0003-1916-9837

Complete contact information is available at: https://pubs.acs.org/10.1021/acsami.0c07532

\section{Author Contributions}

S.R., M.T.V., and M.A.A. contributed equally. The manuscript was written through the contributions of all authors. All authors have given approval to the final version of the manuscript.

\section{Funding}

The authors acknowledge the financial support from King Abdullah University of Science and Technology (KAUST), Saudi Arabia. K.N.S. would like to acknowledge the funding from AMPM Center under the CCF grant. Also, the research leading to these results has received funding from Spanish MINECO and FEDER (projects MAT2016-78257-R and MAT2017-86826-R) and the Aragon Government (DGA) and FEDER (research group E31_17R).

\section{Notes}

The authors declare no competing financial interest.

\section{ACKNOWLEDGMENTS}

M.A.A. acknowledges the support of Ministerio de Educación from the Spanish Government under a FPU grant (Formación de Profesorado Universitario, FPU14/05367) and a short-term mobility FPU grant (EST18/00291) and of King Abdullah University of Science and Technology and Advanced Membranes and Porous Materials Center under the Visiting Student Program. The authors also acknowledge Prof. Dr. Christian Serre for kindly providing MIL-96(Al) NPs used in this contribution.

\section{REFERENCES}

(1) Jia, T. J.; Wang, Y.; Dou, Y. Y.; Li, Y. W.; de Andrade, M. J.; Wang, R.; Fang, S. L.; Li, J. J.; Yu, Z.; Qiao, R.; Liu, Z. J.; Cheng, Y.; Su, Y. W.; Minary-Jolandan, M.; Baughman, R. H.; Qian, D.; Liu, Z. F. Moisture Sensitive Smart Yarns and Textiles from Self-Balanced Silk Fiber Muscles. Adv. Funct. Mater. 2019, 29, No. 1808241.

(2) Wu, J.; Wang, Z.; Liu, W.; Wang, L.; Xu, F. Bioinspired Superelastic Electroconductive Fiber for Wearable Electronics. ACS Appl. Mater. Interfaces 2019, 11, 44735-44741.

(3) Ma, L. Y.; Wu, R. H.; Patil, A.; Zhu, S. H.; Meng, Z. H.; Meng, H. Q.; Hou, C.; Zhang, Y. F.; Liu, Q.; Yu, R.; Wang, J.; Lin, N. B.; Liu, X. Y. Full-Textile Wireless Flexible Humidity Sensor for Human Physiological Monitoring. Adv. Funct. Mater. 2019, 29, No. 1904549.

(4) Guo, R.; Wang, H. M.; Sun, X. Y.; Yao, S. Y.; Chang, H.; Wang, H. Z.; Liu, J.; Zhang, Y. Y. Semiliquid Metal Enabled Highly Conductive Wearable Electronics for Smart Fabrics. ACS Appl. Mater. Interfaces 2019, 11, 30019-30027.

(5) Owyeung, R. E.; Panzer, M. J.; Sonkusale, S. R. Colorimetric Gas Sensing Washable Threads for Smart Textiles. Sci. Rep. 2019, 9, No. 5607.

(6) Smart Textiles Are Fashion's Fourth Industrial Revolution. https://risnews.com/report-smart-textiles-are-fashions-fourthindustrial-revolution (accessed June 03, 2020).

(7) Stoppa, M.; Chiolerio, A. Wearable Electronics and Smart Textiles: A Critical Review. Sensors 2014, 14, 11957-11992.

(8) Fang, X.; Zong, B. Y.; Mao, S. Metal-Organic Framework-Based Sensors for Environmental Contaminant Sensing. Nano-Micro Lett. 2018, 10, 1-19.

(9) Kumar, P.; Deep, A.; Kim, K. H. Metal organic frameworks for sensing applications. TrAC, Trends Anal. Chem. 2015, 73, 39-53.

(10) Zhang, Z. H.; Duan, F. H.; Tian, J. Y.; He, J. Y.; Yang, L. Y.; Zhao, H.; Zhang, S.; Liu, C. S.; He, L. H.; Chen, M.; Chen, D. M.; $\mathrm{Du}, \mathrm{M}$. Aptamer-Embedded Zirconium-Based Metal-Organic Framework Composites Prepared by De Novo Bio-inspired Approach with Enhanced Biosensing for Detecting Trace Analytes. ACS Sens. 2017, 2, 982-989.

(11) Assen, A. H.; Yassine, O.; Shekhah, O.; Eddaoudi, M.; Salama, K. N. MOFs for the Sensitive Detection of Ammonia: Deployment of fcuMOF Thin Films as Effective Chemical Capacitive Sensors. ACS Sens. 2017, 2, 1294-1301.

(12) Sachdeva, S.; Soccol, D.; Gravesteijn, D. J.; Kapteijn, F.; Sudholter, E. J. R.; Gascon, J.; de Smett, L. C. P. M. Polymer-Metal 
Organic Framework Composite Films as Affinity Layer for Capacitive Sensor Devices. ACS Sens. 2016, 1, 1188-1192.

(13) Kim, K. J.; Lu, P.; Culp, J. T.; Ohodnicki, P. R. Metal-Organic Framework Thin Film Coated Optical Fiber Sensors: A Novel Waveguide-Based Chemical Sensing Platform. ACS Sens. 2018, 3, 386-394.

(14) Emam, H. E.; Abdelhameed, R. M. Anti-UV Radiation Textiles Designed by Embracing with Nano-MIL ( $\mathrm{Ti}$, In)-Metal Organic Framework. ACS Appl. Mater. Interfaces 2017, 9, 28034-28045.

(15) Li, G. P.; Cao, F.; Zhang, K.; Hou, L.; Gao, R. C.; Zhang, W. Y.; Wang, Y. Y. Design of Anti-UV Radiation Textiles with SelfAssembled Metal-Organic Framework Coating. Adv. Mater. Interfaces 2020, 7, No. 1901525.

(16) Rubin, H. N.; Neufeld, B. H.; Reynolds, M. M. SurfaceAnchored Metal-Organic Framework-Cotton Material for Tunable Antibacterial Copper Delivery. ACS Appl. Mater. Interfaces 2018, 10, 15189-15199.

(17) López-Maya, E.; Montoro, C.; Rodriguez-Albelo, L. M.; Cervantes, S. D. A.; Lozano-Perez, A. A.; Cenis, J. L.; Barea, E.; Navarro, J. A. R. Textile/Metal-Organic-Framework Composites as Self-Detoxifying Filters for Chemical-Warfare Agents. Angew. Chem., Int. Ed. 2015, 54, 6790-6794.

(18) Lee, D. T.; Zhao, J. J.; Peterson, G. W.; Parsons, G. N. Catalytic "MOF-Cloth" Formed via Directed Supramolecular Assembly of UiO66- $\mathrm{NH}_{2}$ Crystals on Atomic Layer Deposition-Coated Textiles for Rapid Degradation of Chemical Warfare Agent Simulants. Chem. Mater. 2017, 29, 4894-4903.

(19) Smith, M. K.; Mirica, K. A. Self-Organized Frameworks on Textiles (SOFT): Conductive Fabrics for Simultaneous Sensing, Capture, and Filtration of Gases. J. Am. Chem. Soc. 2017, 139, 1675916767.

(20) Tao, C. L.; Ying, Y. M.; Wang, H.; Chen, B.; Zhu, G. P.; Song, Y. J.; Liu, X. G.; Zhao, Z. J.; Shen, L.; Tang, B. Z. Nonwoven Fabric Coated with a Tetraphenylethene-based Luminescent Metal-organic Framework for Selective and Sensitive Sensing of Nitrobenzene and Ammonia. J. Mater. Chem. C 2018, 6, 12371-12376.

(21) Neufeld, M. J.; Harding, J. L.; Reynolds, M. M. Immobilization of Metal-Organic Framework Copper(II) Benzene1,3,5-tricarboxylate (CuBTC) onto Cotton Fabric as a Nitric Oxide Release Catalyst. ACS Appl. Mater. Interfaces 2015, 7, 26742-26750.

(22) Huang, L. Z.; Hu, X. R.; Chi, L. F. Monolayer-Mediated Growth of Organic Semiconductor Films with Improved Device Performance. Langmuir 2015, 31, 9748-9761.

(23) Ariga, K.; Yamauchi, Y.; Mori, T.; Hill, J. P. 25th Anniversary Article: What Can Be Done with the Langmuir-Blodgett Method? Recent Developments and its Critical Role in Materials Science. Adv. Mater. 2013, 25, 6477-6512.

(24) Andrés, M. A.; Vijjapu, M. T.; Surya, S. G.; Shekhah, O.; Salama, K. N.; Serre, C.; Eddaoudi, M.; Roubeau, O.; Gascon, I. Methanol and Humidity Capacitive Sensors Based on Thin Films of MOF Nanoparticles. ACS Appl. Mater. Interfaces 2020, 12, 41554162.

(25) Makiura, R.; Motoyama, S.; Umemura, Y.; Yamanaka, H.; Sakata, O.; Kitagawa, H. Surface Nano-architecture of a MetalOrganic Framework. Nat. Mater. 2010, 9, 565-571.

(26) Andrés, M. A.; Benzaqui, M.; Serre, C.; Steunou, N.; Gascon, I. Fabrication of Ultrathin MIL-96(Al) Films and Study of $\mathrm{CO}_{2}$ Adsorption/Desorption Processes using Quartz Crystal Microbalance. J. Colloid Interface Sci. 2018, 519, 88-96.

(27) Benito, J.; Sorribas, S.; Lucas, I.; Coronas, J.; Gascon, I. Langmuir-Blodgett Films of the Metal-Organic Framework MIL101(Cr): Preparation, Characterization, and $\mathrm{CO}_{2}$ Adsorption Study Using a QCM-Based Setup. ACS Appl. Mater. Interfaces 2016, 8, $16486-16492$.

(28) Park, S. Y.; Kim, Y. H.; Lee, S. Y.; Sohn, W.; Lee, J. E.; Kim, D. H.; Shim, Y. S.; Kwon, K. C.; Choi, K. S.; Yoo, H. J.; Suh, J. M.; Ko, M.; Lee, J. H.; Lee, M. J.; Kim, S. Y.; Lee, M. H.; Jang, H. W. Highly Selective and Sensitive Chemoresistive Humidity Sensors based on
rGO/ $\mathrm{MoS}_{2}$ Van der Waals Composites. J. Mater. Chem. A 2018, 6, $5016-5024$

(29) Davis, R. E.; McGregor, G. R.; Enfield, K. B. Humidity: A Review and Primer on Atmospheric Moisture and Human health. Environ. Res. 2016, 144, 106-116.

(30) Chappanda, K. N.; Chaix, A.; Surya, S. G.; Moosa, B. A.; Khashab, N. M.; Salama, K. N. Trianglamine Hydrochloride Crystals for a Highly Sensitive and Selective Humidity Sensor. Sens. Actuators, B 2019, 294, 40-47.

(31) Yasaei, P.; Behranginia, A.; Foroozan, T.; Asadi, M.; Kim, K.; Khalili-Araghi, F.; Salehi-Khojin, A. Stable and Selective Humidity Sensing Using Stacked Black Phosphorus Flakes. ACS Nano 2015, 9, 9898-9905.

(32) Yang, Z.; Liu, A.; Wang, C.; Liu, F.; He, J.; Li, S.; Wang, J.; You, R.; Yan, X.; Sun, P.; Duan, Y.; Lu, G. Improvement of Gas and Humidity Sensing Properties of Organ-like MXene by Alkaline Treatment. ACS Sens. 2019, 4, 1261-1269.

(33) Kan, H.; Li, M.; Li, H.; Li, C.; Zhou, J.; Fu, C.; Luo, J. T.; Fu, Y. Q. A Novel Quartz-Crystal Microbalance Humidity Sensor based on Solution-Processible Indium Oxide Quantum Dots. RSC Adv. 2019, 9, 38531-38537.

(34) Yan, H. L.; Zhang, L.; Yu, P.; Mao, L. Q. Sensitive and Fast Humidity Sensor Based on A Redox Conducting Supramolecular Ionic Material for Respiration Monitoring. Anal. Chem. 2017, 89, 996-1001.

(35) Kano, S.; Kim, K.; Fujii, M. Fast-Response and Flexible Nanocrystal-Based Humidity Sensor for Monitoring Human Respiration and Water Evaporation on Skin. ACS Sens. 2017, 2, 828-833.

(36) Dai, J.; Zhao, H.; Lin, X.; Liu, S.; Liu, Y.; Liu, X.; Fei, T.; Zhang, T. Ultrafast Response Polyelectrolyte Humidity Sensor for Respiration Monitoring. ACS Appl. Mater. Interfaces 2019, 11, 64836490.

(37) Li, N.; Jiang, Y.; Zhou, C. H.; Xiao, Y.; Meng, B.; Wang, Z. Y.; Huang, D. Z.; Xing, C. Y.; Peng, Z. C. High-Performance Humidity Sensor Based on Urchin-Like Composite of $\mathrm{Ti}_{3} \mathrm{C}_{2}$ MXene-Derived $\mathrm{TiO}_{2}$ Nanowires. ACS Appl. Mater. Interfaces 2019, 11, 38116-38125.

(38) Chappanda, K. N.; Shekhah, O.; Yassine, O.; Patole, S. P.; Eddaoudi, M.; Salama, K. N. The Quest for Highly Sensitive QCM Humidity Sensors: The Coating of CNT/MOF Composite Sensing Films as Case Study. Sens. Actuators, B 2018, 257, 609-619.

(39) Tchalala, M. R.; Belmabkhout, Y.; Adil, K.; Chappanda, K. N.; Cadiau, A.; Bhatt, P. M.; Salama, K. N.; Eddaoudi, M. Concurrent Sensing of $\mathrm{CO}_{2}$ and $\mathrm{H}_{2} \mathrm{O}$ from Air Using Ultramicroporous Fluorinated Metal-Organic Frameworks: Effect of Transduction Mechanism on the Sensing Performance. ACS Appl. Mater. Interfaces 2019, 11, 1706-1712.

(40) Sapsanis, C.; Omran, H.; Chernikova, V.; Shekhah, O.; Belmabkhout, Y.; Buttner, U.; Eddaoudi, M.; Salama, K. N. Insights on Capacitive Interdigitated Electrodes Coated with MOF Thin Films: Humidity and VOCs Sensing as a Case Study. Sensors 2015, $15,18153-18166$.

(41) Wu, J.; Sun, Y. M.; Wu, Z. X.; Li, X.; Wang, N.; Tao, K.; Wang, G. P. Carbon Nanocoil-Based Fast-Response and Flexible Humidity Sensor for Multifunctional Applications. ACS Appl. Mater. Interfaces 2019, 11, 4242-4251.

(42) Guo, H. Y.; Lan, C. Y.; Zhou, Z. F.; Sun, P. H.; Wei, D. P.; Li, C. Transparent, Flexible, and Stretchable $\mathrm{WS}_{2}$ based Humidity Sensors for Electronic Skin. Nanoscale 2017, 9, 6246-6253.

(43) Huang, X.; Li, B.; Wang, L.; Lai, X.; Xue, H.; Gao, J. Superhydrophilic, Underwater Superoleophobic, and Highly Stretchable Humidity and Chemical Vapor Sensors for Human Breath Detection. ACS Appl. Mater. Interfaces 2019, 11, 24533-24543.

(44) Wang, Y.; Zhang, L. N.; Zhou, J. P.; Lu, A. Flexible and Transparent Cellulose-Based Ionic Film as a Humidity Sensor. ACS Appl. Mater. Interfaces 2020, 12, 7631-7638.

(45) Hosseini, Z. S.; Zad, A. I.; Ghiass, M. A.; Fardindoost, S.; Hatamie, S. A New Approach to Flexible Humidity Sensors using Graphene Quantum Dots. J. Mater. Chem. C 2017, 5, 8966-8973. 
(46) Sapsanis, C.; Buttner, U.; Omran, H.; Belmabkhout, Y.; Shekhah, O.; Eddaoudi, M.; Salama, K. N. In A Nafion Coated Capacitive Humidity Sensor on a Flexible PET Substrate, Midwest Symposium on Circuits and Systems (MWSCAS); 2016; pp 321324.

(47) Quddious, A.; Yang, S.; Khan, M. M.; Tahir, F. A.; Shamim, A.; Salama, K. N.; Cheema, H. M. Disposable, Paper-Based, InkjetPrinted Humidity and $\mathrm{H}_{2} \mathrm{~S}$ Gas Sensor for Passive Sensing Applications. Sensors 2016, 16, No. 2073.

(48) Weremczuk, J.; Tarapata, G.; Jachowicz, R. Humidity Sensor Printed on Textile with use of ink-jet Technology. Procedia Eng. 2012, 47, 1366-1369.

(49) Rosace, G.; Trovato, V.; Colleoni, C.; Caldara, M.; Re, V.; Brucale, M.; Piperopoulos, E.; Mastronardo, E.; Milone, C.; De Luca, G.; Plutino, M. R. Structural and Morphological Characterizations of MWCNTs Hybrid Coating onto Cotton Fabric as Potential Humidity and Temperature Wearable Sensor. Sens. Actuators, B 2017, 252, 428-439.

(50) Zhu, P.; Liu, Y.; Fang, Z.; Kuang, Y.; Zhang, Y.; Peng, C.; Chen, G. Flexible and Highly Sensitive Humidity Sensor Based on Cellulose Nanofibers and Carbon Nanotube Composite Film. Langmuir 2019, $35,4834-4842$.

(51) Zhou, G. H.; Byun, J. H.; Oh, Y.; Jung, B. M.; Cha, H. J.; Seong, D. G.; Um, M. K.; Hyun, S.; Chou, T. W. Highly Sensitive Wearable Textile-Based Humidity Sensor Made of High-Strength, Single-Walled Carbon Nanotube/Poly(vinyl alcohol) Filaments. ACS Appl. Mater. Interfaces 2017, 9, 4788-4797.

(52) Shi, H. H.; Jang, S.; Naguib, H. E. Freestanding Laser-Assisted Reduced Graphene Oxide Microribbon Textile Electrode Fabricated on a Liquid Surface for Supercapacitors and Breath Sensors. ACS Appl. Mater. Interfaces 2019, 11, 27183-27191.

(53) Guo, Y. N.; Gao, Z. Y.; Wang, X. X.; Sun, L.; Yan, X.; Yan, S. Y.; Long, Y. Z.; Han, W. P. A Highly Stretchable Humidity Sensor based on Spandex Covered Yarns and Nanostructured Polyaniline. RSC Adv. 2018, 8, 1078-1082.

(54) Li, B. T.; Xiao, G.; Liu, F.; Qiao, Y.; Li, C. M.; Lu, Z. S. A Flexible Humidity Sensor based on Silk Fabrics for Human Respiration Monitoring. J. Mater. Chem. C 2018, 6, 4549-4554.

(55) Wang, L. H.; Tian, M. W.; Zhang, Y. Y.; Sun, F. Q.; Qi, X. J.; Liu, Y. M.; Qu, L. J. Helical Core-Sheath Elastic Yarn-based Dual Strain/Humidity Sensors with MXene sensing layer. J. Mater. Sci. 2020, 55, 6187-6194.

(56) Benoit, V.; Chanut, N.; Pillai, R. S.; Benzaqui, M.; Beurroies, I.; Devautour-Vinot, S.; Serre, C.; Steunou, N.; Maurin, G.; Llewellyn, P. L. A Promising Metal-Organic Framework (MOF), MIL-96(Al), for $\mathrm{CO}_{2}$ Separation under Humid Conditions. J. Mater. Chem. A 2018, 6, 2081-2090.

(57) Mohsen-Nia, M.; Amiri, H.; Jazi, B. Dielectric Constants of Water, Methanol, Ethanol, Butanol and Acetone: Measurement and Computational Study. J. Solution Chem. 2010, 39, 701-708.

(58) Qiang, S.; Carey, T.; Arbab, A.; Song, W.; Wang, C.; Torrisi, F. Wearable Solid-State Capacitors based on Two-Dimensional Material All-Textile Heterostructures. Nanoscale 2019, 11, 9912-9919.

(59) Chmelik, C.; Hibbe, F.; Tzoulaki, D.; Heinke, L.; Caro, J.; Li, J.; Karger, J. Exploring the Nature of Surface Barriers on MOF Zn(tbip) by Applying IR Microscopy in High Temporal and Spatial Resolution. Microporous Mesoporous Mater. 2010, 129, 340-344.

(60) Kreno, L. E.; Leong, K.; Farha, O. K.; Allendorf, M.; Van Duyne, R. P.; Hupp, J. T. Metal-Organic Framework Materials as Chemical Sensors. Chem. Rev. 2012, 112, 1105-1125.

(61) Koo, W. T.; Jang, J. S.; Kim, I. D. Metal-Organic Frameworks for Chemiresistive Sensors. Chem 2019, 5, 1938-1963.

(62) The National Institute of Occupational Safety and Health

(NIOSH). https://www.cdc.gov/niosh/index.htm. 